\title{
ANALYSIS ON THE UTILITY OF SATELLITE IMAGERY FOR DETECTION OF AGRICULTURAL FACILITY
}

\author{
Joon-Mook Kang ${ }^{\mathrm{a}}$, Seung-Hee Baek ${ }^{\mathrm{b}}$, Kap-Yong Jung ${ }^{\mathrm{a}}{ }^{*}$ \\ a Department of Civil Engineering, College of Engineering, Chungnam National University \\ 99 Daehak-ro, Yuseong-gu, Daejeon, ROK - jmkang@cnu.ac.kr, jungjusa@hanmail.net \\ ${ }^{\mathrm{b}}$ Department of Environment \& Ecology Research, Chungnam Development Institute \\ 101 Geumheung-dong, Gongju-si, Chungcheongnam-do, ROK - rgzoa05@nate.com
}

Commission III, WG III/3

KEY WORDS: Satellite Imagery, Agricultural Facility, Image Classification, Formosat-2, Remote Sensing

\begin{abstract}
:
Now that the agricultural facilities are being increase owing to development of technology and diversification of agriculture and the ratio of garden crops that are imported a lot and the crops cultivated in facilities are raised in Korea, the number of vinyl greenhouses is tending upward. So, it is important to grasp the distribution of vinyl greenhouses as much as that of rice fields, dry fields and orchards, but it is difficult to collect the information of wide areas economically and correctly. Remote sensing using satellite imagery is able to obtain data of wide area at the same time, quickly and cost-effectively collect, monitor and analyze information from every object on earth. In this study, in order to analyze the utilization of satellite imagery at detection of agricultural facility, image classification was performed about the agricultural facility, vinyl greenhouse using Formosat-2 satellite imagery. The training set of sea, vegetation, building, bare ground and vinyl greenhouse was set to monitor the agricultural facilities of the object area and the training set for the vinyl greenhouses that are main monitoring object was classified and set again into 3 types according the spectral characteristics. The image classification using 4 kinds of supervise classification methods applied by the same training set were carried out to grasp the image classification method which is effective for monitoring agricultural facilities. And, in order to minimize the misclassification appeared in the classification using the spectral information, the accuracy of classification was intended to be raised by adding texture information. The results of classification were analyzed regarding the accuracy comparing with that of naked-eyed detection. As the results of classification, the method of Mahalanobis distance was shown as more efficient than other methods and the accuracy of classification was higher when adding texture information. Hence the more effective monitoring of agricultural facilities is expected to be available if the characteristics such as texture information including satellite images or spatial pattern are studied in detail.
\end{abstract}

\section{INTRODUCTION}

Now that the agricultural facilities are being increase owing to diversification of agriculture and the ratio of garden crops that are imported a lot and the crops cultivated in facilities are raised in Korea, the number of vinyl greenhouses is tending upward. So, it is important to grasp the distribution of vinyl greenhouses as much as that of rice fields, dry fields and orchards, but it is difficult to collect the information of wide areas economically and correctly. Remote sensing using satellite imagery is able to obtain data of wide area at the same time, quickly and costeffectively collect, monitor and analyze information from every object on earth (Hwang, 2007). In this study, Jeju Island, Korea selected as the study area and the effective classification method is suggested for vinyl greenhouse detection through the image classification of various kinds by using high resolution satellite image.

\section{SUBJECT MATTER AND METHOD}

In the study site Seogwipo, Jeju Island, Korea, agriculture along with tourism business has developed with warm temperate climate, especially as a lot of fruit-growing and tropical fruit cultivation is done, it is an area where the proportion of house plantation is high. Also, with the high profitability of recent facility cultivation, conversion from outdoors cultivation to facility cultivation is actively processed, so it is an area where the continuous grasp of vinyl greenhouse is important. The study site is presented on Fig. 1.

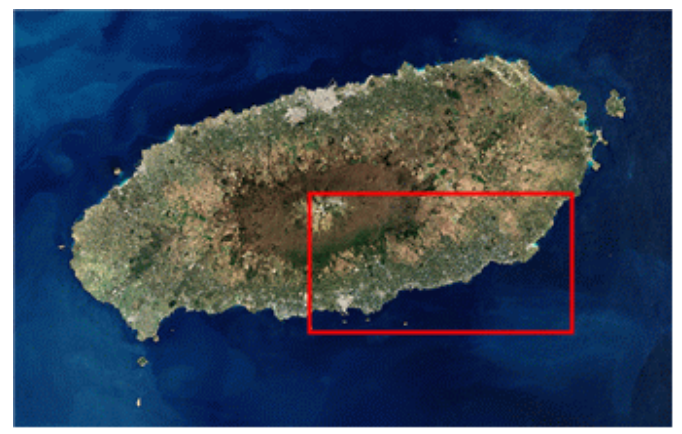

Figure 1. Study Area

This study carried out the supervised classification by the parallel-piped classification, minimum distance classification, maximum likelihood classification, Maharanobis distance classification using the Formosat-2 satellite image of the study

\footnotetext{
* Corresponding author.
} 
site, and the rule-based classification was carried out by using the spatial information and the texture information. In addition, the new classified image was created through the connection by extracting the house plantation in each result of the supervised classification and the result of the rule-based classification. The result of rule-based classification method was masked by the mask band created using the result of supervised classification method.

Each classified image deciphered the classification degree by comparing the visual interpretation results based on the specific areas such as the vegetation area, downtown area etc. In addition, the accuracy was analyzed by comparing between the house plantation area and visual interpretation area of the classification results.

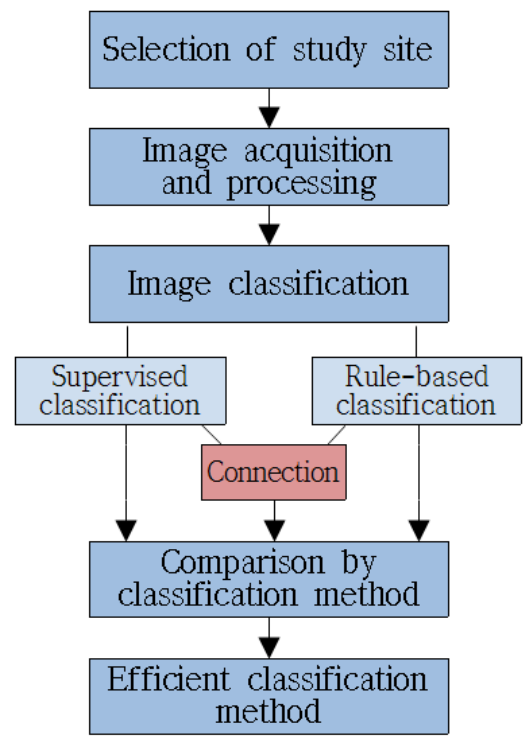

Figure 2. Flow Chart

\section{ANALYSIS OF IMAGE CLASSIFICATION AND RESULT}

For the vinyl greenhouse detection, two copies of Formosat-2 image photographed in March, 20th, April, 8th and 15th 2008 for including all Jeju areas were obtained, and it was treated as mosaic. The mosaic image was divided for including Seogwipo area as the study area, and the vinyl greenhouse was extracted through the image classification.

\subsection{Image Classification}

\section{(1) Supervised classification}

If the user knows the information about the subjects, then the supervised classification method to acquire information about the unknown region based on the information must be effective. For the image classification by the supervised classification, the classification item was set by the sea, vegetation, buildings and bare soil, vinyl greenhouse, and the image classification was carried out by using the parallel-piped classification, minimum distance classification, maximum likelihood classification, Maharanobis distance classification.

\begin{tabular}{|c|c|l|l|}
\hline Characteristic & Symbol & & Class \\
\hline The Sea & Sea & & blue \\
\hline \multirow{2}{*}{ Vegetation } & Forest & & sea green \\
\hline & Grass & & green \\
\hline Buildings & Building & & cyan \\
\hline Bare Soil & Soil & & yellow \\
\hline \multirow{3}{*}{ Vinyl greenhouse } & Vinyl 1 & & red \\
\hline & Vinyl 2 & & magenta \\
\hline & Vinyl 3 & & maroon \\
\hline
\end{tabular}

Table 1. Class of Image Classification

(2) Rule-based classification

The vinyl greenhouse was extracted by using the object-based, rule-based classification. The rule-based classification added the rule to create the rule about attribution for dividing the subjects such as the spatial information and spectral information etc. and to clearly distinguish the subjects (Biao et al., 2007). This study defined the rule to extract the vinyl greenhouse as follows.

- NDVI value is lower than the value of vegetation.

- Form is akin to a rectangle.

- The form is no longer than the road.

- It has the constant area range.

Based on the rule above, the range of the attribute value which can classify the attribution of the vinyl greenhouse most was designated. The designation about the range of the attribute value is based on the user's decision, so the best value should be selected through the various attempts.

\section{(3) Visual interpretation}

In order to be used as the comparison materials for the accuracy analysis of the image classification results, the vinyl greenhouse was extracted through the visual interpretation.

\subsection{RESULT ANALYSIS}

(1) Detection results by the classification method

The results by each classification method were divided with the vegetation area, downtown area, so the misclassification degree and the features were examined.

The image classification results of the parallel-piped classification of the supervised classification method were not good so it was excluded from the subjects of examination.

In the vegetation area with only the vegetation and vinyl greenhouse, the classification of the vinyl greenhouse was carried out in the classification method used in the study. But in the rule-based classification, the minority of the objects of the vinyl greenhouse was not extracted. In the downtown area, the misclassification was found from all classification method to take part in. The building with the similar vinyl greenhouse and spectral information was misclassified with the vinyl greenhouse in the supervised classification method, and Maharanobis distance classification showed the best results. In the case of the rule-based classification, the vegetation, bare soil, building with the similar form of the object, vegetation index and the size with the vinyl greenhouse was misclassified as the vinyl greenhouse. Fig. 3 showed the classification results in the downtown area. 


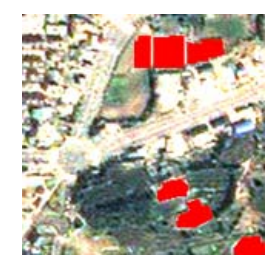

(a) Visual Interpretation

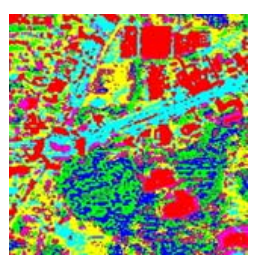

(b) Minimum Distance Classification

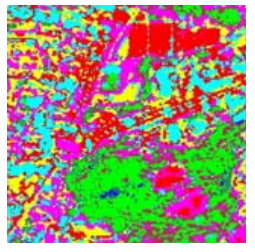

(c) Maximum

Likelihood

Classification

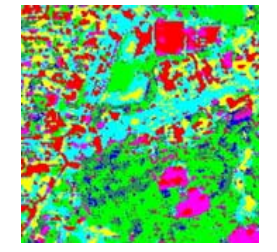

(d) Mahalanobis

Distance

Classification

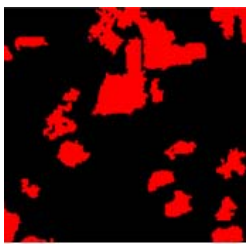

(e) rule-based classification
Figure 3. Classification result in downtown area

In the case of the supervised classification method, the classification about the vegetation, bare soil parts were carried out well, but the building parts with similar spectral information with the vinyl greenhouse showed the misclassification. But on the other hand, the similar vegetation, bare soil parts of the object attribute with the vinyl greenhouse showed misclassification except the building by the rule-based classification method. It means the misclassification features with different features, so the vinyl greenhouse was extracted by connection of the method, rule-based classification method in each supervised classification. Mask band was created using greenhouse which was extracted in supervised classification result. Vinyl greenhouse corresponding with result of rulebased classification was masked using the created mask band. This helped to obtain new classification image.

As the results of the connection, the vegetation area showed overall, good extract results, but the parts which were missed in the rule-based classification were not included in the connection process also. In the downtown area, a considerable number of building parts which were misclassified due to the spectral information in the supervised classification method was reduced through the connection process, and the vegetation and bare soil parts which were misclassified with the vinyl greenhouse were removed in the rule-based classification results. But some parts which were misclassified from the supervised classification method and rule-based classification were not removed. Fig. 4 showed the connected classification results in the downtown area.

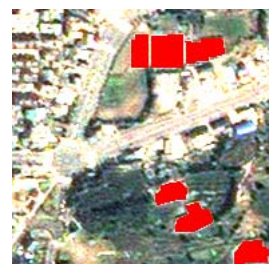

(a) Visual Interpretation

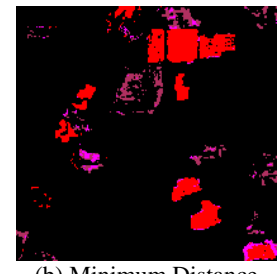

(b) Minimum Distance Classification

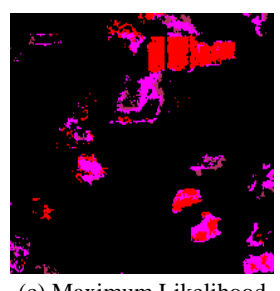

(c) Maximum Likelihood Classification

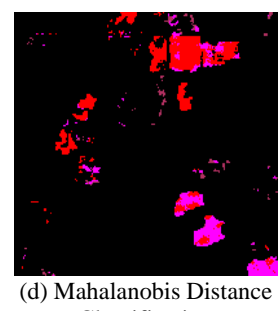

Classification

\section{Figure 4. Connection classification results} in the downtown area

(2) Analysis on the land cover detection accuracy

For the land cover detection accuracy analysis, the Maharanobis distance classification which the vinyl greenhouse was classified most among the supervised classification methods was selected, and the accuracy analysis was carried out by comparing with the visual interpretation results. The accuracy analysis was carried out by calculating the distribution area of the vinyl greenhouse by using the area of each pixel. Fig. 5 showed the area rate graph by each classification method based on the visual interpretation area.

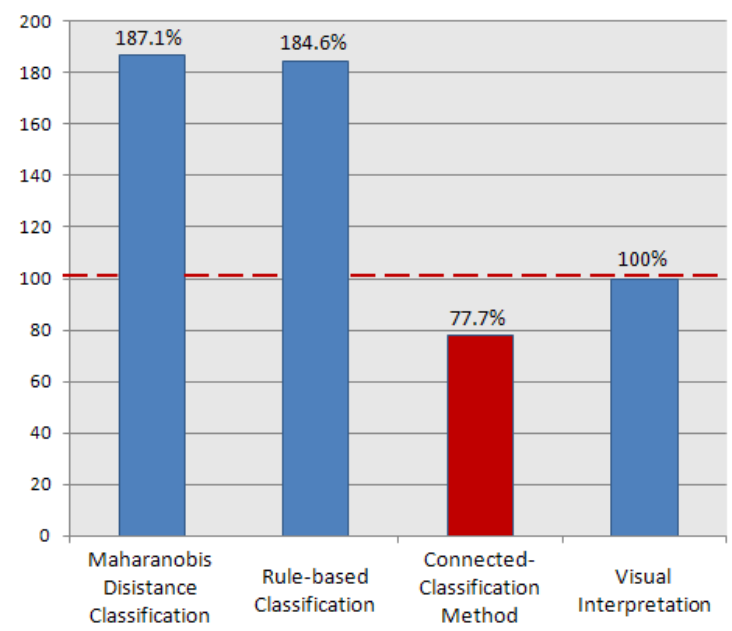

Figure 5. Vinyl greenhouse distribution area by classification method

If the visual interpretation area is $100 \%$, Maharanobis distance classification is $187.1 \%$, rule-based classification is $184.6 \%$, and it can be seen that the vinyl greenhouse was over-classified than the existing distribution. In the case of Maharanobis distance classification, it is judged that the object with the similar spectral information was misclassified with the vinyl greenhouse, and in the case of the rule-based classification method, the attribution of the object such as the vegetation figure, form, size etc. is similar with the rule dividing the vinyl greenhouse and it is misclassified. In addition, it can be assumed that the quantity of misclassification is significantly reduced with $77 \%$ of visual interpretation areas for the connection of two methods, and it is judged that each part which was misclassified in the connection process was carried out with complementary. 


\section{CONCLUSIONS}

This study aims to carry out the image classification by using the Formosat-2 satellite image and to suggest the effective classification method for the vinyl greenhouse detection through the accuracy comparison by the image classification method.

The parallel-piped classification, minimum distance classification, maximum likelihood classification, Maharanobis distance classification among the supervised classification methods and rule-based classification were carried out, and the proper classification method for the vinyl greenhouse detection was assumed. In addition, the misclassification item of two classification methods was treated with complementary by creating the image after connecting the results of the supervised classification method and the rule-based classification. It can be seen when Maharanobis distance classification and rule-based classification were connected through the area comparison of the results of each classification method and visual interpretation detection, the vinyl greenhouse detection accuracy can be improved. It is expected that it can be used effectively for the vinyl greenhouse detection if the missing parts in the connection process of the supervised classification method and rule-based classification method can be complemented in the future.

\section{REFERENCES}

Biao Oin, Yuni Xia, Sunil Prabhakar, Yicheng Tu, 2009, A Rule-Based Classification Algorithm for Uncertain Data, Proceedings of the 2009 IEEE International Conference on Data Engineering, pp. 1633-1640.

Chen, H. W., Chang, N. B., Yu, R. F., Huang, Y. W., 2009, Urban land use and land cover classification using the neuralfuzzy inference approach with Formosat-2 data, Journal of applied remote sensing, Vol. 3, No. 1, 033558.

Cho, E. R., Kim, K. W., Yoo, H. H., 2009, Analysis of Land Use Change Using High Resolution Satellite Imagery, Journal of the Korean Society for Geospatial Information System, 17(1), pp. 3-11.

Hwang, H. H., 2007, The Accuracy Estimation of Landcover Map for Reliability, Thesis of Master, Kyonggi University, pp. $1-66$.

Lee, J. Y., Kim, B. S., 2008, Automated Green House Extraction Method Using Texture Information in High Spatial Resolution Satellite Image, Proceeding of annual spring conference of the Korean Society of Remote Sensing, pp. 48-52.

Li, Z., Hayward, R, Zhang, J., Jin, H., Walker, R., 2010, Evaluation of Spectral and Texture Features for Object-Based Vegetation Species Classification Using Support Vector Machines, ISPRS TC VII Symposium Vienna - 100 Years ISPRS, IAPRS, Vol. XXXVIII, Part 7A, pp.122-127.

Oh, C. Y., Park, S. Y., Kim, H. S., Lee, Y. W., Choi, C. U., 2010, Comparision of Landcover Map Accuracy Using High Resolution Satellite Imagery, Journal of the Korean Association of Geographic Information Studies, 13(1), pp. 89100. 